\title{
The direct effect of leptin on skeletal muscle thermogenesis is mediated by substrate cycling between de novo lipogenesis and lipid oxidation
}

\author{
Giovanni Solinas $^{\mathrm{a}, \mathrm{b}}$, Serge Summermatter ${ }^{\mathrm{a}}$, Davide Mainieri ${ }^{\mathrm{a}}$, Marcel Gubler ${ }^{\mathrm{c}}$, Luciano Pirola ${ }^{\mathrm{d}}$, \\ Matthias P. Wymann ${ }^{b}$, Sandro Rusconi ${ }^{\mathrm{b}}$, Jean-Pierre Montani ${ }^{\mathrm{a}}$, Josiane Seydoux ${ }^{\mathrm{e}}$, \\ Abdul G. Dulloo, ${ }^{\mathrm{a}, *}$ \\ ${ }^{a}$ Department of Medicine, Division of Physiology, University of Fribourg, Switzerland \\ ${ }^{\mathrm{b}}$ Department of Medicine, Division of Biochemistry, University of Fribourg, Switzerland \\ ${ }^{\mathrm{c}}$ Department of Vascular and Metabolic Diseases, Hoffmann-La Roche, Switzerland \\ ${ }^{\mathrm{d}}$ Faculty of Medicine, INSERM, Nice, France \\ ${ }^{\mathrm{e}}$ Department of Physiology, Faculty of Medicine, University of Geneva, Switzerland
}

Received 18 October 2004; accepted 27 October 2004

Available online 4 November 2004

Edited by Vladimir Skulachev

\begin{abstract}
We report here studies that integrate data of respiration rate from mouse skeletal muscle in response to leptin and pharmacological interference with intermediary metabolism, together with assays for phosphatidylinositol 3-kinase (PI3K) and AMP-activated protein kinase (AMPK). Our results suggest that the direct effect of leptin in stimulating thermogenesis in skeletal muscle is mediated by substrate cycling between de novo lipogenesis and lipid oxidation, and that this cycle requires both PI3K and AMPK signaling. This substrate cycling linking glucose and lipid metabolism to thermogenesis provides a novel thermogenic mechanism by which leptin protects skeletal muscle from excessive fat storage and lipotoxicity.

(C) 2004 Federation of European Biochemical Societies. Published by Elsevier B.V. All rights reserved.
\end{abstract}

Keywords: Obesity; Diabetes; Lipotoxicity; Gluco-lipotoxicity; Insulin resistance; Phosphatidylinositol 3-kinase; AMP-activated protein kinase; Sterol regulatory element binding protein-1c

\section{Introduction}

Skeletal muscle, which accounts for $30-40 \%$ of body mass in mammals, is an important site for glucose disposal, lipid oxidation and thermogenesis whose impairments contribute to the pathogenesis of obesity and type 2 diabetes. It has long been suspected that these metabolic events are often interdependent in normal and disease states [1,2], but a mechanistic link between glucose and lipid metabolism to skeletal muscle thermogenesis is still ill-defined. Leptin, an adipocyte-derived hormone which is well known for its role in weight regulation,

\footnotetext{
${ }^{*}$ Corresponding author. Fax: +41-26-300-9734.

E-mail address: abdul.dulloo@unifr.ch (A.G. Dulloo).
}

Abbreviations: $\mathrm{MO}_{2}$, respiration (oxygen consumption) rate; EDL, extensor digitorum longus; PI3K, phosphatidylinositol 3-kinase; AMPK, AMP-activated protein kinase; ObRb, long form of the leptin receptor; PY, phosphotyrosine; IRS1, insulin receptor substrate 1; IRS2, insulin receptor substrate 2; CPT-1, carnitine palmitoyl transferase-1; ACC, acetyl-CoA carboxylase; araA, adenine 9- $\beta$-D-arabinofuranoside; AICAR, 5-aminoimidazole-4-carboxamide ribonucleoside has also been shown to protect insulin-sensitive tissues like skeletal muscle against excessive fat storage that can lead to functional impairments known as lipotoxicity [3]. The demonstrations that leptin can act directly on skeletal muscle, specifically via the long form of the leptin receptor $(\mathrm{ObRb})$, to stimulate glucose utilization [4], lipid oxidation through AMPactivated protein kinase (AMPK) [5,6] or thermogenesis in a phosphatidylinositol 3-kinase (PI3K)-dependent manner [7], have provided the impetus to investigate the mechanisms by which muscle substrate metabolism and thermogenesis are interdependent. Although the mechanisms leading to increased fatty acid oxidation in skeletal muscle in response to leptin have been described in molecular details [6], those underlying its effects on thermogenesis are still unknown, amid continuing controversies concerning the role of novel uncoupling proteins, UCP2 and UCP3, as effectors of skeletal muscle thermogenesis $[3,8,9]$. Furthermore, the mechanism by which glucose and lipid metabolism are linked to thermogenesis in response to leptin's direct effect on skeletal muscle is unknown. With the objective of elucidating the mechanisms by which leptin exerts its direct effect on skeletal muscle thermogenesis, we report here a study that integrates data of respiration rate from intact mouse skeletal muscle ex vivo in response to leptin and pharmacological interference with key control points of intermediary metabolism, together with biochemical measurements for PI3K and AMPK signaling.

\section{Materials and methods}

\subsection{Mice and muscle tissue preparations}

Intact muscles were obtained from 7 to 8 week old male BALB/ cByJIco mice (Charles River Laboratories, L'Arbresle, France). For ex vivo calorimetric measurements, soleus and/or extensor digitorum longus (EDL) muscles were carefully dissected out intact together with their tendons and freed of loosely attached connective tissue. They were then placed on a stainless steel frame, at physiological resting length, in the test chambers of a twin indirect microcalorimeters perifused with Krebs-Ringer bicarbonate buffer at $30^{\circ} \mathrm{C}$, as described previously [7].

\subsection{Measurement of tissue respiration rate}

The respiratory rate $\left(\mathrm{MO}_{2}\right)$ of skeletal muscle was measured by a method involving repeated $\mathrm{O}_{2}$ uptake determinations, as described by 
Barde et al. [10]. The $\mathrm{O}_{2}$ partial pressure of a bubble-free liquid phase enclosed in a thick-walled Lucite chamber was measured by a Clark $\mathrm{O}_{2}$ electrode connected to a polarographic circuit, whose output voltage is directly proportional to the $\mathrm{O}_{2}$ partial pressure. At about $10 \mathrm{~min}$ intervals, a peristaltic pump partially exchanges the solution for a fresh one within 2-3 min. All values for $M_{2}$ were taken during steady-state respiration. For each hormone or drug, this corresponded to $90-120$ min after administration. The basal steady state $M \mathrm{O}_{2}$ was taken 120 $150 \mathrm{~min}$ after placing the muscle preparations in the experimental chambers.

\subsection{AMPK Thr172 phosphorylation, PI3K assays and leptin receptor antibodies}

Soleus and EDL muscles were incubated in the presence of leptin $(10 \mathrm{nM})$ or insulin $(100 \mathrm{nM})$ or AICAR $(10 \mathrm{mM})$ for $15 \mathrm{~min}$, and then immediately frozen in liquid nitrogen. Frozen muscles were homogenized and incubated in lysis buffer $(20 \mathrm{mM}$ Tris- $\mathrm{HCl}, 138$ $\mathrm{mM} \mathrm{NaCl}, 2.7 \mathrm{mM} \mathrm{KCl}, 5 \%(\mathrm{v} / \mathrm{v})$ glycerol, NP-40, and protease inhibitors) for $15 \mathrm{~min}$. After centrifugation at $13000 \mathrm{rpm}$ for $15 \mathrm{~min}$, protein concentration was quantified and the protein extracts were used for measurement of AMPK Thr172 phosphorylation or PI3K activity. For AMPK phosphorylation, $200 \mu \mathrm{g}$ of protein extract was immunoprecipitated with phospho-AMPK $\alpha$ (Thr172) polyclonal antibodies (Cell Signalling). The samples were then separated on a $10 \%$
SDS-PAGE gel and blotted on a PVDF membrane that was analyzed with same phospho-AMPK $\alpha$ (Thr172) polyclonal antibodies. For PI3K assay, $200 \mu \mathrm{g}$ of protein extract was immunoprecipitated either with phosphotyrosine (PY) monoclonal antibody (Cell Signalling), with p85 rabbit polyclonal antibody (obtained from MP Wymann laboratory), with insulin receptor substrate 1 (IRS1) polyclonal antibody (Cell Signalling), or with insulin receptor substrate 2 (IRS2) polyclonal antibody (Upstate). The kinase reaction, thin-layer chromatography separation and signal detection were performed as described by Pirola et al. [11]. Western blot analysis for the $\mathrm{ObRb}$ was made using anti-leptin receptor polyclonal antibody (Affinity BioReagents Inc).

\subsection{Muscle de novo lipogenesis analysis}

Pools of four left leg muscles and four right leg muscles from the same animals were incubated separately in buffer containing $5 \mathrm{mM}$ glucose supplemented with $\mathrm{D}-\left[{ }^{14} \mathrm{C}\right]$-glucose for control and insulin treatment reactions, respectively. After incubation for $2 \mathrm{~h}$ at $30^{\circ} \mathrm{C}$, muscle lipids were extracted and separated by thin-layer chromatography on silica gel developed with hexane:ethylether:acetic acid 80:20:1. ${ }^{14} \mathrm{C}$-labeled lipid metabolites were detected by phosphor imaging and compared with lipid standards, namely: ${ }^{14} \mathrm{C}$-labeled palmitate and mixture of unlabeled mono-, di-, and tri-oleoylglycerol that were detected colorimetrically.
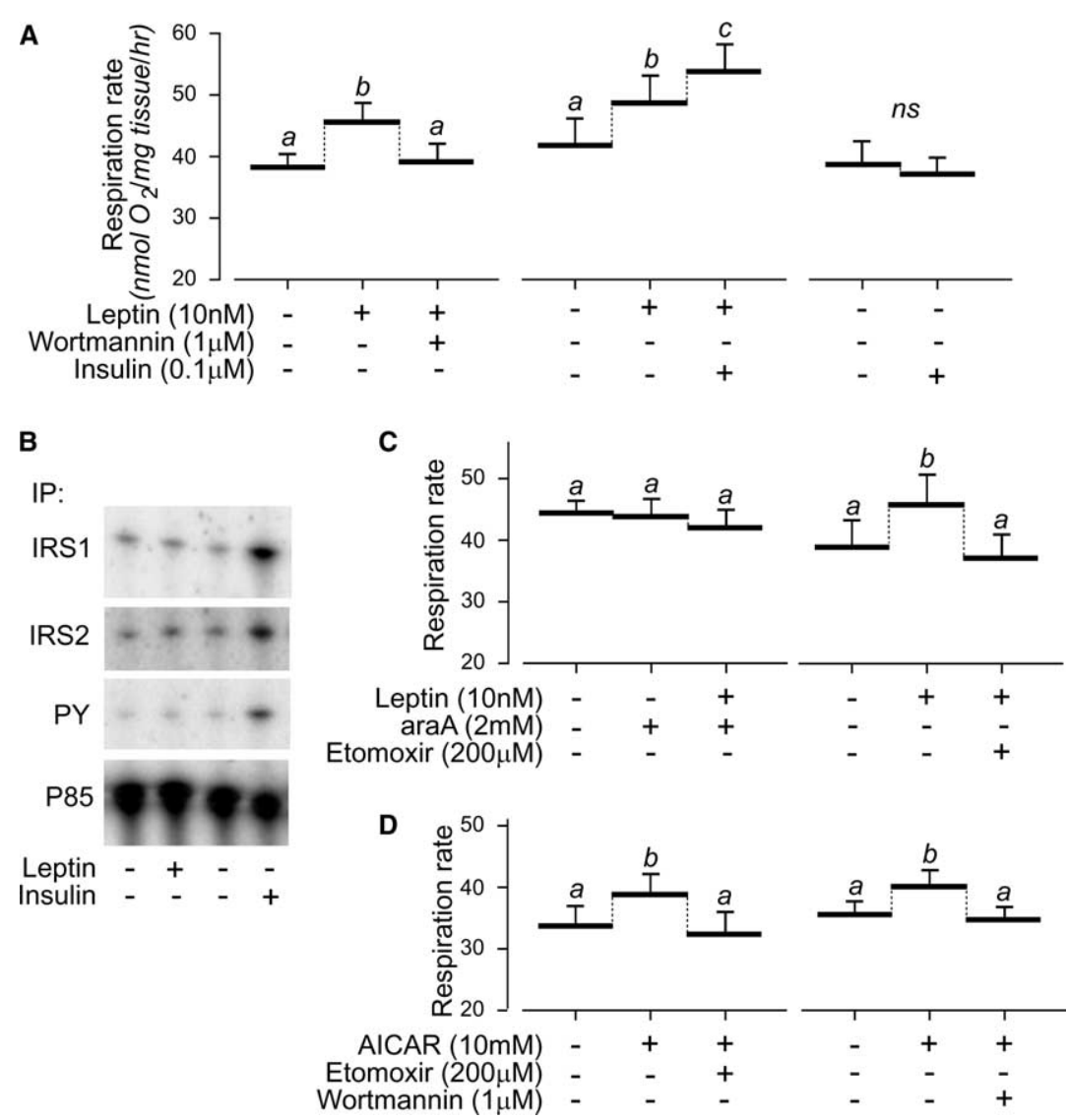

Fig. 1. (A) Steady-state respiration rate $\left(\mathrm{MO}_{2}\right)$ of soleus muscles in the basal state, during sequential addition of leptin and wortmannin (an inhibitor of PI3K), or during sequential addition of leptin and insulin; Note that addition of insulin alone had no significant effect on basal $M \mathrm{O}_{2}$; (B) IRS1, IRS2, PY and p85 associated PI3K activity in response to leptin or to insulin; (C) $M \mathrm{O}_{2}$ of soleus muscles in the basal state, during the sequential addition of araA (a competitive inhibitor of AMPK) and leptin, or during the sequential addition of leptin and the CPT-1 inhibitor, etomoxir; (D) $\mathrm{MO}_{2}$ of soleus muscles in the basal state, during sequential addition of AICAR and etomoxir, or during sequential addition of AICAR and wortmannin. It is to be noted that addition of etomoxir alone or wortmannin alone had no significant effect on basal $M \mathrm{O}_{2}$ (data not shown). For data on $\mathrm{MO}_{2}$, values are means $(n=5-6)$, with vertical bars representing standard errors. Significant effects were assessed by ANOVA with repeated measures. Following ANOVA, post hoc pairwise comparisons were made by Tukey's test and values not sharing the same superscript (a-c) are significantly different from each other $(P<0.05)$; ns $=$ no significant difference. For the PI3K assay, comparisons between presence and absence of leptin or between presence and absence of insulin were made between pools of four left leg soleus muscles and four right leg soleus muscles from the same animals, and the experiment was replicated three times. 


\subsection{Chemicals and drugs}

All chemicals were purchased from Fluka (Buchs, Switzerland). Recombinant murine leptin was purchased from Insight Biotechnology Ltd. (Middlesex, UK), Wortmannin and Hydroxy-citrate from Calbiochem (Luzern, Switzerland), Cerulenin from Fluka (Buchs, Switzerland), 5-aminoimidazole-4-carboxamide ribonucleoside (AICAR) from Toronto Research Chemicals (TRC, Toronto, Canada) and Adenine 9- $\beta$-D-arabinofuranoside (araA) from Sigma (St. Louis, MO, USA). Etomoxir was a generous gift from Prof. W. Langhans (Zurich, Switzerland).

\section{Results}

\subsection{Requirement for PI3K signaling}

Consistent with our previous report [7], leptin $(10 \mathrm{nM})$ stimulates the respiration rate $\left(M \mathrm{O}_{2}\right)$ of soleus muscle (by about $20 \%$ ), and this effect is abolished by the addition of wortmannin, an inhibitor of PI3K (Fig. 1A). Since leptin induces associations of PI3K activity to PY residues and to IRS1 and IRS2 in muscle myotubes [12] and in skeletal muscle from mice injected with leptin [13], we investigate here whether an increase in PI3K association with these molecules also correlates with the direct effect of leptin on muscle $M \mathrm{O}_{2}$. Using in vitro kinase assays in ex vivo intact soleus muscles incubated with leptin, insulin or saline solution as control, we however found that there is no induction of PY, IRS1, IRS2 and p85 associated PI3K activities in response to leptin, in contrast to what is observed in response to insulin (Fig. 1B). Our results therefore suggest that PI3K requirement for leptin-mediated thermogenesis in skeletal muscle is independent of an increase in PI3K association to IRS1, IRS2 and PY as well as to an increase of $\mathrm{p} 85$ associated pool. Considering that the calorimetric data show that leptin's stimulation of $\mathrm{MO}_{2}$ in soleus muscle is PI3K dependent, it is possible that leptin induces PI3K associations to molecules other than those investigated, or that leptin does not stimulate PI3K activity in muscle but that basal levels of PI3K activity (inhibited by wortmannin) are required to support the direct thermogenic effect of leptin. Consistent with a role for PI3K in leptin-induced thermogenesis in muscle is the finding that insulin, which activates PI3K signaling in our ex vivo muscle preparations (Fig. 1B), enhances the stimulatory effect of leptin on $\mathrm{MO}_{2}$ in soleus muscle (Fig. 1A). However, since stimulation of PI3K activity by insulin per se failed to produce an increase in $\mathrm{MO}_{2}$ (Fig. 1A), we conclude that the direct thermogenic effect of leptin in soleus muscle requires additional signaling pathway(s).

\subsection{Requirement for AMPK-ACC-CPT-1 axis and PI3K signaling}

It is now established that leptin can directly stimulate fatty acid oxidation at the expense of fatty acid storage in soleus muscle [5], and that this effect is mediated via leptin's stimu-

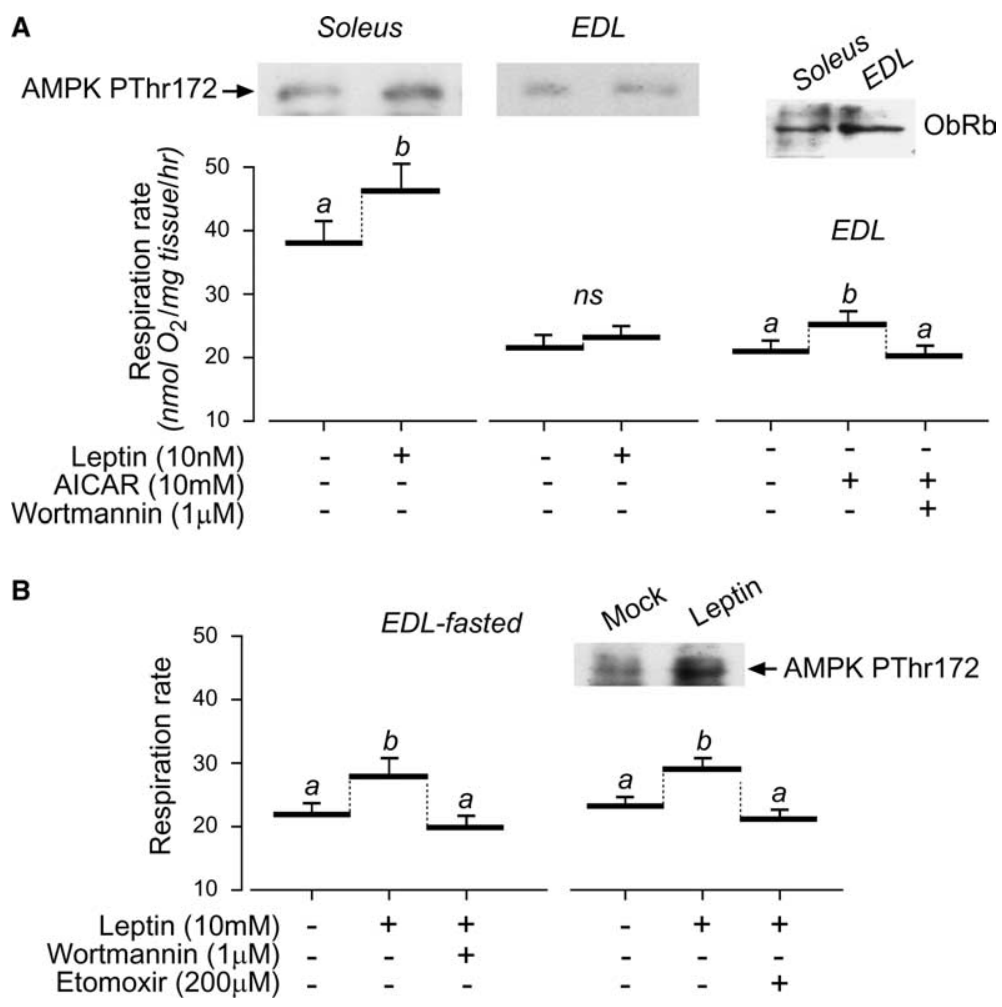

Fig. 2. (A) AMPK phosphorylation and respiration rate $\left(M \mathrm{O}_{2}\right)$ of soleus and EDL muscles from ad libitum fed mice in response to leptin; note that in EDL muscle the lack of increases in $M \mathrm{O}_{2}$ and in AMPK in response to leptin is not explained by a lower protein expression of the ObRb. This panel also shows that the increase in $M \mathrm{O}_{2}$ of EDL muscle from ad libitum fed mice in response to AICAR is inhibited in the presence of wortmannin; (B) data are obtained on EDL muscles from $16 \mathrm{~h}$-fasted mice for $M \mathrm{O}_{2}$ in the basal state, during sequential addition of leptin and wortmannin, or during sequential addition of leptin and etomoxir. All data on $M \mathrm{O}_{2}$ are means, with vertical bars representing standard errors $(n=5-7)$; values not sharing the same superscript $(\mathrm{a}, \mathrm{b})$ are significantly different from each other $(P<0.05)$. For the AMPK assay, pools of four left leg muscles (soleus or EDL) and four right leg muscles (soleus or EDL) from the same animals were incubated in the presence or absence of leptin, and the experiment was replicated three times; For the ObRb assay, comparisons were made between pools of four soleus muscles and four EDL muscles from the left leg of the same animals, and the experiment was also replicated three times. 
lation along the axis of AMPK $\rightarrow$ acetyl-CoA carboxylase (ACC) $\rightarrow$ carnitine palmitoyl transferase-1 (CPT-1) [6]. To test the role of this axis in leptin-mediated thermogenesis, we investigated whether pharmacological inhibition of AMPK activation or CPT-1 leads to an inhibition of the effect of leptin on muscle $\mathrm{MO}_{2}$. We show here that prior addition of araA, an intracellular competitive inhibitor of AMPK [14], prevents leptin-induced increases in muscle $M \mathrm{O}_{2}$, and that etomoxir, a CPT-1 inhibitor in muscle [15], blunts the direct effect of leptin on muscle $\mathrm{MO}_{2}$ (Fig. 1C). To further elucidate the role of the AMPK-ACC-CPT-1 axis, and to determine whether activation of AMPK alone is sufficient to increase skeletal muscle thermogenesis, soleus muscles were treated with AICAR, an activator of AMPK [16]. We show (Fig. 1D) that AICAR stimulates $M \mathrm{O}_{2}$ in soleus muscles and that this effect is blocked not only by etomoxir but also by wortmannin. Taken together, these data suggest that, in addition to stimulation of AMPKACC-CPT-1 axis, a basal level of PI3K is also necessary for the direct effects of AICAR and leptin on skeletal muscle thermogenesis.

Further support that both PI3K signaling and AMPK activation are necessary for the direct effects of leptin and AICAR on muscle thermogenesis can be derived from studies, described below, that utilize either the soleus muscle and/or the EDL muscle. Since an increase in AMPK activity in response to leptin was previously reported [6] to occur only in soleus (a predominantly slow-oxidative fiber type) but not in EDL (a predominantly fast-glycolytic fiber type), we investigated whether this muscle-specific activation of AMPK signaling correlates with leptin-induced thermogenesis. We show here that, in contrast to soleus muscle, the addition of leptin to EDL muscle, whether at the concentration of $10 \mathrm{nM}$ (Fig. 2A) or at $100 \mathrm{nM}$ (data not shown), does not significantly increase either $\mathrm{MO}_{2}$ or AMPK phosphorylation, thereby underscoring the association between AMPK stimulation and muscle thermogenesis in response to leptin. Since we found that this lack of EDL responsiveness to leptin cannot be explained by a lower expression of the ObRb (Fig. 2A), we postulated that it could be consequential to post-receptor signaling defect. To test whether the absence of thermogenic response to leptin in the EDL can be attributed to a lack of AMPK activation, we used AICAR which has been shown to directly stimulate AMPK activity in this tissue [6]. The addition of AICAR to EDL muscle increased $M_{2}$ (Fig. 2A), which suggests that the unresponsiveness of EDL to leptin is uniquely due to a lack of AMPK activation. This AICAR-induced increase in $M \mathrm{O}_{2}$ in EDL is also inhibited by addition of the PI3K inhibitor, wortmannin (Fig. 2A), which is consistent with the above observations in soleus muscle, and further supports the requirement for PI3K activity in the direct effect of AICAR and leptin on skeletal muscle $\mathrm{MO}_{2}$.

Since during fasting, substrate oxidation in glycolytic muscles shifts from glucose to fatty acids accompanied by elevated CPT-1 activity, the question arose as to whether EDL could have the potential to respond to leptin in muscles from fasted mice. Indeed, in EDL muscles from 16h fasted mice, leptin was effective in increasing both $\mathrm{MO}_{2}$ and AMPK phosphorylation, and these increases in $M \mathrm{O}_{2}$ were also found to be inhibited by the PI3K inhibitor, wortmannin, and by the CPT-1 inhibitor, etomoxir (Fig. 2B). Taken together, these studies from soleus and EDL muscles underscore the requirement for basal PI3K

activity and AMPK activation in the direct thermogenic effect of leptin in skeletal muscle.

\subsection{Requirement for glucose and de novo lipogenesis}

Our findings using the CPT-1 inhibitor etomoxir (Figs. 1C and $2 \mathrm{~B}$ ) show that fatty acid entry into the mitochondrial $\beta$ oxidation is necessary for the thermogenic effect of leptin in muscle. Since leptin can also stimulate glucose uptake and metabolism in soleus muscle [4], we investigated whether glucose metabolism is also necessary for the thermogenic response to leptin. We show here (Fig. 3A) that the direct effect of leptin on $M \mathrm{O}_{2}$ in soleus muscle is inhibited by the replacement of glucose in the perifusion medium with 2-deoxyglucose, a glucose analog whose metabolism stops after its phosphorylation by hexokinase, thereby indicating that leptin-induced thermogenesis in muscle requires not only fatty acid oxidation but also glucose metabolism. These data, together with the fact that PI3K - which in addition to controlling glucose uptake and its conversion to lipids (i.e., de novo lipogenesis) in several cell types [17] - is also required for muscle thermogenesis in response to leptin, led us to test the following hypothesis, namely that de novo lipogenesis can occur in skeletal muscle and, in response to leptin, glucose will first be converted to lipids before being oxidized in muscle mitochondria. This would generate an energy dissipating 'futile' cycle that can be an effector of the thermogenic effects of leptin.

A
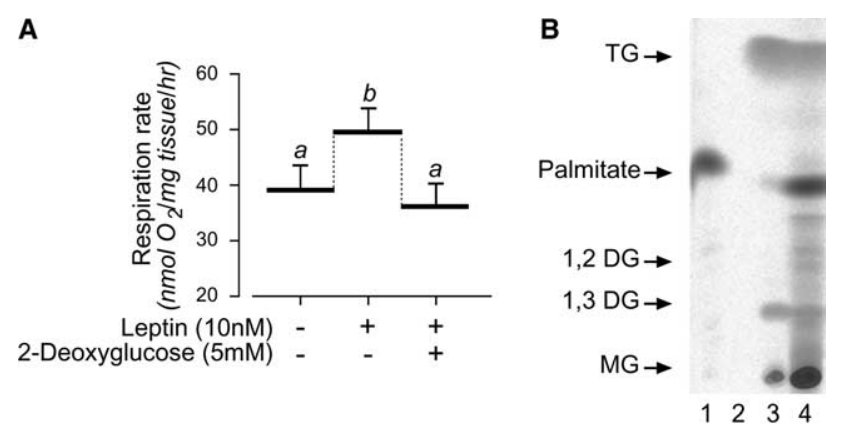

C

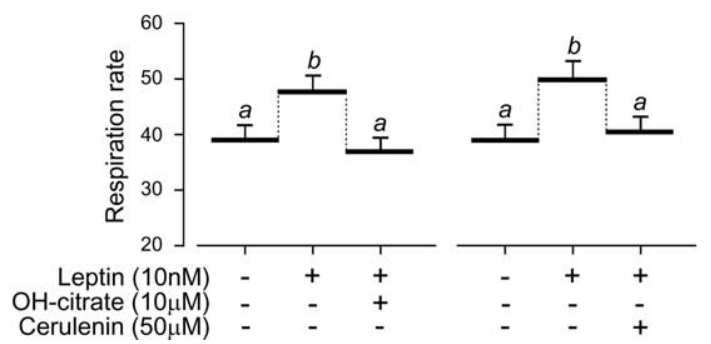

Fig. 3. (A) Respiration rate $\left(M \mathrm{O}_{2}\right)$ of soleus muscle in the basal state, during addition of leptin, and subsequently after addition of leptin in buffer in which glucose has been replaced by 2-deoxyglucose; (B) Phosphor imaging of thin layer chromatography showing conversion of $\mathrm{D}-\left[{ }^{14} \mathrm{C}\right]$-glucose into $\left[{ }^{14} \mathrm{C}\right]$-lipids in mouse soleus muscle: lane 3 contains lipids extracted from muscles in control buffer and lane 4 contains lipids extracted from muscles incubated with $100 \mathrm{nM}$ insulin; lipid standards are as follows: lane $1:{ }^{14} \mathrm{C}$-labeled palmitate and lane 2, mixture of unlabeled mono-(MG), di-(DG) and tri-oleoylglycerol (TG) that were detected colorimetrically; $(\mathrm{C}) \mathrm{MO}_{2}$ in the basal state, during sequential addition of leptin and $\mathrm{OH}$-citrate, an inhibitor of citrate lyase, or during sequential addition of leptin and cerulenin, an inhibitor of fatty acid synthase. All data are means, with vertical bars representing standard errors $(n=6-8)$; values not sharing the same superscript $(\mathrm{a}, \mathrm{b})$ are significantly different from each other $(P<0.05)$. 
We show here that incubation of our muscle preparations in buffer containing ${ }^{14} \mathrm{C}$-labeled glucose resulted in the production of ${ }^{14} \mathrm{C}$-labeled lipids corresponding to free fatty acids, diacylglycerol and triacylglycerol when analyzed by thin-layer chromatography (Fig. 3B), and that insulin increased the synthesis of lipids from glucose in soleus muscle, and to a lesser extent in EDL muscles (data not shown). This experiment demonstrates that de novo lipogenesis can occur in skeletal muscle and that it can be induced by insulin, as in the liver and adipose tissue [17]. To know whether de novo lipogenesis is necessary for leptin-induced thermogenesis in skeletal muscle, we tested whether leptin-induced increases in $\mathrm{MO}_{2}$ in the soleus muscle are inhibited by the addition of inhibitors of key control points in the conversion of glucose to lipids. We show here that $\mathrm{OH}$-citrate, which inhibits the enzyme citrate lyase, or cerulenin, an inhibitor of fatty acid synthase, can blunt the leptin induction of $\mathrm{MO}_{2}$ in soleus muscle (Fig. 3C). Taken together, these data indicate that both glucose metabolism and de novo lipogenesis are required for the direct thermogenic effect of leptin in skeletal muscle.

\section{Discussion}

Here, we show that the direct effect of leptin on skeletal muscle thermogenesis requires both de novo lipogenesis and lipid oxidation, as well as both AMPK and PI3K signaling. These results suggest that the direct stimulatory effect of leptin on thermogenesis in skeletal muscle is dependent upon substrate cycling between de novo lipogenesis and lipid oxidation. It is postulated that in this substrate cycle, which underscores the interdependency between glucose metabolism, lipid oxidation and thermogenesis, acetyl-CoA produced from glucose and fatty acid oxidation will overload the Krebs cycle (Fig. 4). This will result in excess mitochondrial citrate which, in the cytoplasm, will exert an allosteric activation of the enzyme ACC and at the same time, under the action of citrate lyase, will provide acetyl-CoA to ACC for the synthesis of malonylCoA. The latter will serve as the main substrate for fatty acid synthase, thereby producing a new pool of fatty acids. Glucose plays a central role in this cycle as a source of acetyl-CoA, Krebs cycle intermediates and NADPH molecules, which are required for the synthesis of free fatty acids. It might also function as a stimulator of de novo lipogenesis, based upon the recent demonstration [18] in rat muscle satellite cells that glucose, even in the absence of insulin, stimulates sterol regulatory element binding protein-1c (SREBP-1c) gene expression as well as key genes encoding glycolytic and lipogenic enzymes, leading to an increased lipogenic flux and intracellular lipid accumulation. The latter study [18] also provides direct evidence that de novo lipogenesis can occur in skeletal muscle cells.

AMPK and PI3K signaling could orchestrate this 'futile' cycle between de novo fatty acid synthesis and fatty acid oxidation. On the one hand, activation of AMPK, by phosphorylating ACC, will counterbalance the stimulatory action of citrate on ACC to result in reduced malonyl-CoA concentration, disinhibition of CPT-1 and increased fatty acid oxidation, which in turn will lead to the production of acetylCoA and consequently overloading of the Krebs cycle. On the other hand, PI3K activity (basal or insulin-stimulated)

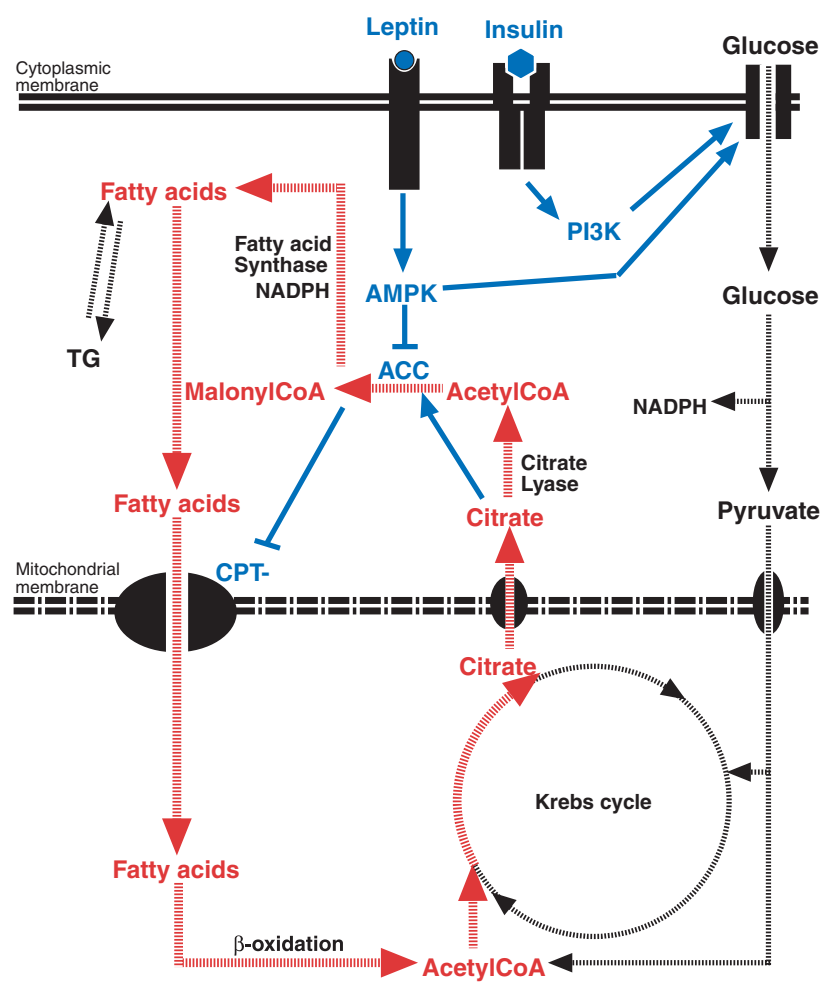

Fig. 4. Model illustrating an energy-dissipating 'futile' substrate cycling between de novo lipogenesis and lipid oxidation, orchestrated by PI3K and AMPK signaling, in skeletal muscle (see text for details). TG: triglycerides; CPT-1: carnitine palmitoyl transferase-1; ACC: acetyl-CoA carboxylase.

will increase glucose entry and allow the excess citrate to enter pathways leading to the synthesis of fatty acids. This would occur despite AMPK-induced reduction in malonyl$\mathrm{CoA}$, since it is known that full phosphorylation of ACC by AMPK results in an inhibition of ACC activities only by 50 $60 \%[19,20]$. Such partial inhibition of ACC is expected to redirect the flux of acetyl-CoA and malonyl-CoA towards fatty acid oxidation, but would still allow substantial rate of fatty acid synthesis, particularly in the presence of high levels of citrate.

This substrate cycling between de novo lipogenesis and lipid oxidation therefore constitutes a thermogenic effector in skeletal muscle. Theoretically, the synthesis of one molecule of palmitic acid from acetyl-CoA and its re-oxidation to acetyl-CoA would cost at least 14 molecules of ATP. The evidence supporting the existence of this substrate cycling as a thermogenic effector in muscle rests upon our data on metabolic labeling in intact skeletal muscle showing the occurrence of de novo lipogenesis (Fig. 3B), and upon our calorimetric data showing that the direct thermogenic effects of leptin in skeletal muscle are inhibited by interference with key control points in this flux of substrates (Fig. 4), namely: (i) with glucose metabolism using 2-deoxyglucose, (ii) with the conversion of citrate to acetyl-CoA using $\mathrm{OH}$-citrate, an inhibitor of citrate lyase, (iii) with the conversion of malonyl-CoA to fatty acids using cerulenin, an inhibitor of fatty acid synthase, and (iv) with entry of fatty acid into mitochondrial $\beta$-oxidation pathway using etomoxir, an inhibitor of CPT-1. 
Furthermore, evidence that the rate of this substrate cycle could be orchestrated by PI3K and AMPK signaling rests upon our data here showing that: (a) leptin-mediated thermogenesis requires CPT-1 which is the final effector of the leptin-AMPK-ACC-CPT-1 axis, (b) the thermogenic effect of leptin is inhibited by pharmacological inhibition of AMPK activation, (c) a strong correlation exists between AMPK activation and thermogenesis in soleus and/or EDL muscles in response to leptin, (d) the thermogenic effects of leptin is inhibited by the PI3K inhibitor, wortmannin, and (e) insulin, a stimulator of PI3K activity, potentiates the thermogenic effects of leptin on skeletal muscle and induces de novo lipogenesis as shown from our metabolic labeling experiments. In fact, a dual requirement for AMPK and PI3K signaling is strongly supported by our findings that the administration of insulin alone does not stimulate muscle thermogenesis (Fig. 1A), and that the activation of AMPK by AICAR leads to an increase in thermogenesis that is abolished by the PI3K inhibitor wortmannin (Fig. 1D).

This energy dissipating substrate cycling linking glucose and lipid metabolism to thermogenesis provides a novel molecular mechanism by which leptin protects the skeletal muscle from ectopic fat storage and lipotoxicity. Although the increase in muscle metabolic rate induced by leptin (about 20\%) may not, a priori, be considered to be large, it must be emphasized that peripheral resistance to this thermogenic effect of leptin can, over time, lead to the accumulation of intramyocellular lipids or lipid moieties, which even in modest amounts, can lead to insulin resistance and lipotoxicity [3]. Approaches that enhance this energy-dissipating substrate cycle in skeletal muscle may thus have therapeutic value for obesity and type 2 diabetes.

Acknowledgements: We thank Dorothée Rohrer for excellent technical support and Prof. Wolfgang Langhans (Zurich) for his generous gift of etomoxir. This work was supported by Grant Nos. 3157129.991 and 3200B0-102156 of the Swiss National Science Foundation.

\section{References}

[1] Randle, P.J., Garland, P.B., Hales, C.N. and Newsholme, E.A. (1963) The glucose-fatty acid cycle: its role in insulin sensitivity and metabolic disturbances of diabetes mellitus. Lancet I, 785789.

[2] Newsholme, E.A. (1980) A possible metabolic basis for the control of body weight. N. Engl. J. Med. 302, 400-405.

[3] Unger, R.H. and Orci, L. (2001) Diseases of liporegulation: new perspective on obesity and related disorders. FASEB J. 15, 312321 .

[4] Ceddia, R.B., William Jr., W.N. and Curi, R. (2001) The response of skeletal muscle to leptin. Front. Biosci. 6, D90-97.

[5] Muoio, D.M., Dohn, G.L., Fiedorek, F.T., Tapscott, E.B. and Coleman, R.A. (1997) Leptin directly alters lipid partitioning in skeletal muscle. Diabetes 46, 1360-1363.
[6] Minokoshi, Y., Kim, Y.B., Peroni, O.D., Fryer, L.G., Muller, C., Carling, D. and Kahn, B.B. (2002) Leptin stimulates fatty-acid oxidation by activating AMP-activated protein kinase. Nature 415, 339-343.

[7] Dulloo, A.G., Stock, M.J., Solinas, G., Boss, O., Montani, J.P. and Seydoux, J. (2002) Leptin directly stimulates thermogenesis in skeletal muscle. FEBS Letters 515, 109-113.

[8] Samec, S., Seydoux, J. and Dulloo, A.G. (1998) Role of UCP homologues in skeletal muscles and brown adipose tissue: mediators of thermogenesis or regulators of lipids as fuel substrate? FASEB J. 12, 715-724.

[9] Goglia, F. and Skulachev, V.P. (2003) A function for novel uncoupling proteins: antioxidant defense of mitochondrial matrix by translocating fatty acid peroxides from the inner to the outer membrane leaflet. FASEB J. 17, 1585-1591.

[10] Barde, Y., Chinet, A. and Girardier, L. (1975) Potassium-induced increase in oxygen consumption of brown adipose tissue from the rat. J. Physiol. (Lond.) 252, 523-536.

[11] Pirola, L., Zvelebil, M.J., Bulgarelli-Leva, G., Van Obberghen, E., Waterfield, M.D. and Wymann, M.P. (2001) Activation loop sequences confer substrate specificity to phosphoinositide 3-kinase alpha (PI3Kalpha). Functions of lipid kinase-deficient PI3Kalpha in signalling. J. Biol. Chem. 276, 21544-21554.

[12] Kellerer, M., Koch, M., Metzinger, E., Mushack, J., Capp, E. and Haring, H.U. (1997) Leptin activates PI-3 kinase in C2C12 myotubes via janus kinase-2 (JAK-2) and insulin receptor substrate-2 (IRS-2) dependent pathways. Diabetologia 40, 13581362.

[13] Maroni, P., Bendinelli, P. and Piccoletti, R. (2003) Early intracellular events induced by in vivo leptin treatment in mouse skeletal muscle. Mol. Cell. Endocrinol. 201, 109-121.

[14] Wu, X., Motoshima, H., Mahadev, K., Stalker, T.J., Scalia, R. and Goldstein, B.J. (2003) Involvement of liver AMP-activated protein kinase in glucose uptake stimulated by the globular domain of adiponectin in primary rat adipocytes. Diabetes 52, $1355-1363$.

[15] Kler, R.S., Sherratt, H.A.S. and Turnbull, D.M. (1992) The measurement of mitochondrial $\beta$ oxidation by release of $3 \mathrm{H} 2 \mathrm{O}$ from $[9,10-3 \mathrm{H}]$ hexadecanoate: application to skeletal muscle and the use of inhibitors as models of metabolic disease. Biochem. Med. Metab. Biol. 47, 145-156.

[16] Corton, J.M., Gillespie, J.G., Hawley, S.A. and Hardie, D.G. (1995) 5-Aminoimidazole-4-carboxamide ribonucleoside. A specific method for activating AMP-activated protein kinase in intact cells. Eur. J. Biochem. 229, 558-565.

[17] Foufelle, F., Girard, J. and Ferre, P. (1996) Regulation of lipogenic enzyme expression by glucose in liver and adipose tissue: a review of the potential cellular and molecular mechanisms. Adv. Enzyme Regul. 36, 199-226.

[18] Guillet-Deniau, I., Pichard, A.L., Koné, A., Esnous, C., Nieruchalski, M., Girard, J. and Prip-Buus, C. (2004) Glucose induces de novo lipogenesis in rat muscle satellite cells through a srerolregulatory-element-binding-protein-1c-dependent pathway. J. Cell Sci. 177, 1937-1944.

[19] Winder, W.W. and Hardie, D.G. (1996) Inactivation of acetylCoA carboxylase and activation of AMP-activated protein kinase in muscle during exercise. Am. J. Physiol. 270, E299-E304.

[20] Gubler, M., Westerberg, R., Andjelkovic, M. and Mizrahi, J. (2003). Functional characterization f human ACC2 enzyme and elucidation of its role in the regulation of mitochondrial fatty acid oxidation. Keystone Conference on Obesity: New insights into pathogenesis and treatment, Keystone: Abstract 214 . 